\title{
Estudo retrospectivo dos procedimentos anestésicos realizados em cães e gatos submetidos a neurocirurgias
}

\section{Retrospective study of anesthetic proceedings realized in dogs and cats undergoing neurosurgeries}

\author{
Daniella Aparecida Godoi ${ }^{1}$; Mariana Isa Poci Palumbo Antunes ${ }^{2}$; \\ Mônica Vicky Bahr Arias; ; Carmen Esther Grumadas; \\ Angelita Zanata Reia ${ }^{4}$; Julio Ken Nagashima ${ }^{5}$
}

\section{Resumo}

\begin{abstract}
Neurocirurgias são cada vez mais freqüentes na rotina dos hospitais veterinários, sendo necessário o conhecimento dos diferentes protocolos anestésicos para tais procedimentos, com a finalidade de evitar morbidade e mortalidade no período trans e pós-cirúrgico. Os objetivos deste trabalho foram avaliar os protocolos anestésicos utilizados em pacientes submetidos a procedimentos cirúrgicos neurológicos; os resultados obtidos com o uso destes protocolos; a taxa de complicações e se existiu relação entre sua ocorrência e o tempo de anestesia. Foram analisadas as fichas anestésicas de 52 cães e dois gatos operados entre jan/ 2003 e dez/ 2006, no Hospital Veterinário da UEL. Através da análise dos resultados, observou-se que os principais protocolos utilizados foram propofol para indução e halotano ou isofluorano para manutenção da anestesia. Estes protocolos mantiveram o plano anestésico desejado para o procedimento cirúrgico e não causaram complicações durante o período anestésico e pós-operatório imediato em 37 dos 54 casos (68,5\%). Entre as complicações observadas, 7/19 (36,8\%) ocorreram em pacientes submetidos à anestesia com halotano e $8 / 32(25,2 \%)$ em pacientes anestesiados com isofluorano. A principal complicação neste estudo foi bradicardia, que acometeu 15/54 (27,8\%) dos pacientes. Ocorreram dois óbitos em pacientes submetidos à técnica de "slot" cervical. É essencial o conhecimento das doenças neurológicas e das técnicas cirúrgicas e anestésicas para evitar alterações no sistema nervoso central, causadas pelos fármacos, pela doença ou associação destes fatores.
\end{abstract}

Palavras-chave: Anestesia, neurocirurgia, complicações, cães, gatos

\begin{abstract}
Neurosurgeries are frequent in the routine of veterinary hospitals and, therefore, knowledge of the different anesthetic protocols to be used for each patient is necessary to prevent the morbity and mortality in the after and trans-surgical period. The objectives of this study were to evaluate the anesthetic protocols used in patients undergoing neurosurgeries; the results of those protocols; the rate of complications, and if those complications are related to the duration of the anesthesia time. We studied the anesthetical data of 52 dogs and two cats submitted to neurosurgery between January of 2003 and December of 2006, in the Veterinary Hospital of UEL. Findings showed that the main protocols used were propofol
\end{abstract}

\footnotetext{
Residente Clínica Médica de Pequenos Animais - Hospital Veterinário - Universidade Estadual de Londrina, Londrina, PR. Autor para correspondência - Av. São Paulo 550 apt 1704, Londrina, PR - e-mail: daniellagodoi@hotmail.com

2 Residente Clínica Médica de Pequenos Animais - Hospital Veterinário - Universidade Estadual de São Paulo, Botucatu, SP.

3 Professora Doutora - Departamento de Clínicas Veterinárias - Universidade Estadual de Londrina.

4 Médica Veterinária - Hospital Veterinário - Universidade Estadual de Londrina.

5 Médico Veterinário Autônomo.

* Autor para correspondência
} 
for induction and halothane or isofluorane for maintenance of the anesthesia. These protocols induced adequate anesthesia for the surgical procedure and did not occur complications during the anesthetic period in $37 / 54(68,5 \%)$ of the cases. $7 / 19(36.8 \%)$ of the complications observed occurred in patients submitted to anesthesia with halothane and $8 / 32(25.2 \%)$ in patients with isofluorane. The complication most observed in this study was bradicardy, which occurred in 15/54 (27.8\%) of the patients. Two deaths occurred in patients submitted to "slot" cervical. Good knowledge of the neurological disease and of surgical and anesthetic techniques are essential to prevent alterations in the central nervous system caused by the drugs, disease or association of these factors.

Key words: Anesthesia, neurosurgery, complications, dogs, cats

\section{Introdução}

Os cães e gatos acometidos por doenças neurológicas muitas vezes necessitam de intervenções cirúrgicas (COURT et al., 1990a). Segundo Seim III (2002), as neurocirurgias realizadas mais freqüentemente em medicina veterinária são para descompressão ou estabilização da coluna vertebral. Além dessas cirurgias, as craniotomias também podem ser realizadas para descompressão do tecido nervoso, biópsias ou excisão de neoplasias (COURT et al., 1990b; SEIM III, 2005). A anestesia geral é essencial para o manejo cirúrgico desses pacientes. Os fármacos utilizados nas anestesias gerais alteram a atividade elétrica neuronal e o suprimento sangüíneo ao tecido nervoso, principalmente em pacientes com doenças neurológicas, nos quais estas funções estão alteradas. Otimizar as técnicas anestésicas nesses pacientes, para minimizar esses efeitos, pode ter impacto marcante no resultado da cirurgia (STEFFEY, 1996), pois o controle anestésico de pacientes com alterações neurológicas requer preservação da função neural, evitando-se hipóxia, hipercapnia, além de instabilidade respiratória e cardiovascular (CORNICK, 1992; SEIM III, 2005), com o objetivo de minimizar a isquemia no sistema nervoso central (CRUZ, 2002).

O fluxo sangüíneo medular responde a alterações na pressão arterial e $\mathrm{PaCo} 2$ de forma análoga à regulação do fluxo sangüíneo cerebral. Em estudo feito por Kobrine, Doyle e Rizzoli (1976), verificouse que a perfusão medular foi reduzida em 50\% quando a pressão arterial diminuiu para $50 \mathrm{mmHg}$, sendo que ao atingir o valor de $30 \mathrm{mmHg}$ a perfusão cessou. Nos traumas medulares ocorre a perda da regulação química e de pressão do fluxo sangüíneo medular (TORRES; BONASSA, 2002). As lesões medulares podem interromper as conexões de tratos nervosos simpáticos advindos do tronco encefálico, diminuindo as funções motoras cardiovasculares, sendo muito importante a escolha correta dos protocolos anestésicos (COURT et al., 1990a; SEIM III, 2005). Alguns anestésicos inalatórios causam alterações cardiovasculares, deprimindo diretamente o miocárdio em maior ou menor grau (SEIM III, 2005; SILVA et al., 2006).

As mudanças fisiológicas induzidas pelo trauma craniano agudo que podem ter impacto na anestesia incluem hipoventilação, hipóxia, hipertensão, taquicardia, arritmias e hipertensão intracraniana. O manejo anestésico desses pacientes deve ser direcionado para melhorar a oxigenação arterial e a função cardiovascular, melhorando a dinâmica intracraniana (TORRES; BONASSA, 2002). Seim III (2005) afirma que em pacientes com trauma crânio-encefálico, o uso de alguns fármacos pode agravar o estado clínico e neurológico do paciente, aumentando a pressão intracraniana e levando à isquemia cerebral.

Os objetivos deste trabalho foram realizar uma avaliação crítica dos protocolos anestésicos utilizados em 54 pacientes (52 cães e dois gatos) submetidos a procedimentos cirúrgicos neurológicos realizados entre janeiro de 2003 a dezembro de 2006 no Hospital Veterinário da Universidade Estadual de Londrina; avaliar os resultados obtidos com o uso destes protocolos e se mantiveram o plano anestésico desejado para o procedimento realizado, a taxa de complicações (óbito e bradicardia) e se existiu relação entre sua ocorrência e o tempo de anestesia. 


\section{Material e Métodos}

O estudo foi realizado de forma retrospectiva, avaliando-se as fichas anestésicas dos pacientes submetidos a neurocirurgias no Hospital Veterinário da Universidade Estadual de Londrina, no período de Janeiro de 2003 a Dezembro de 2006. Foram avaliados os protocolos anestésicos utilizados em 54 pacientes (52 cães e dois gatos) submetidos a procedimentos cirúrgicos, os resultados obtidos com o uso destes protocolos e as complicações ocorridas. O peso dos animais variou entre $0,8 \mathrm{e}$ $47,5 \mathrm{~kg}$, e a idade entre 40 dias e 16 anos (idade média de 6 anos). Dos 54 animais 26 eram fêmeas. As raças presentes neste estudo foram as seguintes: Teckel (22), SRD (6 cães e 2 gatos), Lhasa Apso (5), Cocker (5), Poodle (5), Pinsher (2), Basset Hound (1), Pitt Bull (1), Rottweiler (1), Pequinês (1), Beagle (1), Fila Brasileiro (1) e Pastor Alemão (1).

Estes animais foram divididos em grupos de acordo com a técnica realizada: 42 cães submetidos à hemilaminectomia toracolombar e seis cães ao "slot" cervical para tratamento da doença de disco intervertebral, dois cães e dois gatos à estabilização vertebral (devido à wobbler, fraturas e luxações) e dois cães à craniotomia para retirada de projétil balístico e para descompressão devido à perfuração por mordida de outro cão. Em cada grupo foram avaliados: o tempo de anestesia, os procedimentos anestésicos, o agente inalatório utilizado e a ocorrência de complicações.

Para análise estatística foi utilizado o Teste Exato de Fisher, com nível de significância 5\% $(\alpha=0,05)$, para associar os fármacos utilizados durante as neurocirurgias em cães e gatos (medicação transoperatória e anestésicos inalatórios) às complicações observadas. O Teste t Student, com nível de significância $5 \%(\alpha=0,05)$, foi utilizado para o cálculo de diferença de médias em relação ao tempo anestésico e as complicações ocorridas.

\section{Resultados}

Em 37/54 animais anestesiados (68,5\%) o protocolo utilizado manteve o plano anestésico desejado durante todo o procedimento cirúrgico, não ocorrendo complicações como óbito e bradicardia. Em 17/54 animais (31,5\%) ocorreu alguma dessas complicações durante o procedimento. O tempo médio de cirurgia foi de 2 horas e 34 minutos nos pacientes submetidos à hemilaminectomia (mínimo de 1 hora e 30 minutos e máximo de 4 horas) e de 2 horas e 36 minutos ao "slot" cervical (mínimo de 2 horas e máximo de 3 horas). Nos animais submetidos à estabilização vertebral o tempo médio foi de 3 horas e 10 minutos (mínimo de 2 horas e 40 minutos e máximo de 4 horas e 10 minutos). A craniotomia em um animal durou 1 hora e 40 minutos e no outro, 2 horas e 30 minutos. Através da análise estatística, não houve associação significativa entre o tempo anestésico e as complicações ocorridas $(p=0,092)$.

Levomepromazina foi utilizada como medicação pré-anestésica $(0,15 \mathrm{mg} / \mathrm{kg}$ IV) em 16 animais $(29,6 \%)$, três animais $(5,6 \%)$ receberam a associação de morfina e levomepromazina, e em quatro animais $(7,4 \%)$ utilizou-se diazepam. Os outros 29 animais $(53,7 \%)$ já vinham recebendo analgesia com opióides (morfina) durante o período pré-operatório (Tabela 1). A indução anestésica foi realizada com propofol $(57,4 \%)$ ou propofol associado ao diazepam (37,03\%). Tiopental foi utilizado para indução de dois animais $(3,7 \%)$ e um animal $(1,85 \%)$ foi induzido na máscara com sevofluorano (Tabela 2). 
Tabela 1. Medicações pré-anestésicas (MPA) utilizadas em cães e gatos submetidos a diferentes neurocirurgias realizadas no Hospital Veterinário da UEL, Londrina, 2007.

\begin{tabular}{|c|c|c|c|c|c|}
\hline \multicolumn{6}{|c|}{ MPA } \\
\hline Fármaco & $\mathrm{H}$ & $\mathrm{S}$ & $\mathrm{E}$ & $\mathrm{C}$ & Total \\
\hline Levomepromazina & $13(30,95 \%)$ & $3(50 \%)$ & - & - & $16(29,6 \%)$ \\
\hline Levo + Morfina & $3(7,14 \%)$ & - & - & - & $3(5,6 \%)$ \\
\hline Diazepam & $4(9,52 \%)$ & - & - & - & $4(7,4 \%)$ \\
\hline Morfina & $22(52,38 \%)$ & $3(50 \%)$ & $4(100 \%)$ & - & $29(53,7 \%)$ \\
\hline Total & 42 & 6 & 4 & 2 & 54 \\
\hline
\end{tabular}

H: Hemilaminectomia; S: "Slot”Cervical; E: Estabilização vertebral; C: Craniotomia

Tabela 2. Fármacos indutores utilizados em cães e gatos submetidos a diferentes neurocirurgias realizadas no Hospital Veterinário da UEL, Londrina, 2007.

\begin{tabular}{|c|c|c|c|c|c|}
\hline \multicolumn{6}{|c|}{ Indução } \\
\hline Fármaco & $\mathrm{H}$ & $\mathrm{S}$ & E & $\mathrm{C}$ & Total \\
\hline Propofol & $28(66,67 \%)$ & $1(16,67 \%)$ & $2(50 \%)$ & - & $31(57,4 \%)$ \\
\hline Prop +Diazepam & $14(33,33 \%)$ & $4(66,67 \%)$ & $2(50 \%)$ & - & $20(37,03 \%)$ \\
\hline Sevofluorano (máscara) & - & - & - & $1(50 \%)$ & $1(1,85 \%)$ \\
\hline Tiopental & - & $1(16,67 \%)$ & - & $1(50 \%)$ & $2(3,7 \%)$ \\
\hline Total & 42 & 6 & 4 & 2 & 54 \\
\hline
\end{tabular}

H: Hemilaminectomia; S: "Slot”Cervical; E: Estabilização vertebral; C: Craniotomia

Todos os animais foram submetidos à anestesiados com halotano e quatro com isofluorano. manutenção anestésica com halogenados durante Dentre os quatro pacientes submetidos à técnica de o período transoperátorio (Tabela 3). Dentre os 42 estabilização vertebral, dois foram anestesiados cães submetidos à hemilaminectomia, $14(33,3 \%)$ com isofluorano e dois com halotano. Dentre os foram anestesiados com halotano, $26(61,9 \%)$ com cães submetidos a craniotomia, em um filhote de isofluorano e dois $(4,76 \%)$ com sevoflurano. Para 40 dias com trauma craniano grave utilizou-se a técnica de "slot" cervical, dois animais foram sevofluorano, e no outro, adulto, halotano.

Tabela 3. Agentes inalatórios utilizados para manutenção anestésica de cães e gatos submetidos a neurocirurgias realizadas no Hospital Veterinário da UEL, Londrina, 2007.

\begin{tabular}{|c|c|c|c|c|c|}
\hline \multicolumn{6}{|c|}{ Manutenção } \\
\hline Fármaco & $\mathrm{H}$ & $\mathrm{S}$ & $\mathrm{E}$ & $\mathrm{C}$ & Total \\
\hline Isofluorano & $26(61,9 \%)$ & $4(66,67 \%)$ & $2(50 \%)$ & - & $32(59,26 \%)$ \\
\hline Halotano & $14(33,33 \%)$ & $2(33,33 \%)$ & $2(50 \%)$ & $1(50 \%)$ & $19(35,18 \%)$ \\
\hline Sevofluorano & $2(4,76 \%)$ & - & - & $1(50 \%)$ & $3(5,55 \%)$ \\
\hline Total & 42 & 6 & 4 & 2 & 54 \\
\hline
\end{tabular}

H: Hemilaminectomia; S: “Slot”Cervical; E: Estabilização vertebral; C: Craniotomia 
Todos os animais submetidos à técnica de "slot" cervical foram mantidos com ventilação controlada, e somente dois animais submetidos à hemilaminectomia foram mantidos com respiração espontânea. Dois procedimentos de estabilização vertebral e um de craniotomia também foram realizados sob ventilação controlada.
Dentre os 32 animais anestesiados com isofluorano, 13 cães $(40,6 \%)$ receberam ainda infusão contínua de fármacos analgésicos durante o transoperatório. Destes 13 animais, seis $(46,15 \%)$ receberam infusão contínua de fentanil $(3 \mu \mathrm{g} / \mathrm{kg} / \mathrm{h})$ durante a realização de hemilaminectomia e sete $(53,85 \%)$ receberam infusão contínua de lidocaína $(100 \mu \mathrm{g} / \mathrm{kg} / \mathrm{min})$, sendo seis animais submetidos à hemilaminectomia e um a slot Cervical. (Tabela 4).

Tabela 4. Fármacos utilizados em cães e gatos durante as neurocirurgias realizadas no Hospital Veterinário da UEL e as complicações observadas durante o procedimento anestésico, Londrina, 2007.

\begin{tabular}{cccc}
\hline & \multicolumn{3}{c}{ Medicação Transoperatória } \\
\hline & Iso+F IC & Iso+L IC & Total \\
\hline Bradicardia & $1(16,7 \%)$ & $2(28,6 \%)$ & $3(27,1 \%)$ \\
Óbito & - & $1(14,3 \%)$ & $1(7,7 \%)$ \\
Sem complicações & $5(83,3 \%)$ & $4(57,1 \%)$ & $9(69,2 \%)$ \\
\hline Total & 6 & 7 & 13 \\
\hline
\end{tabular}

Iso: Isofluorano; F: Fentanil; L: Lidocaína; C: Cetamina; IC: Infusão contínua.

Não houve diferença significativa $(\mathrm{p}=1,0)$ quanto à medicação transoperatória e presença de bradicardia Não houve diferença significativa $(p=1,0)$ quanto à medicação transoperatória e presença de óbito

As complicações observadas estão nas tabelas 4 e 5 . Entre os animais anestesiados com halotano $7 / 19$ cães apresentaram bradicardia (36,84\%). Dos animais anestesiados com isofluorano $8 / 32$ (25\%) apresentaram tal complicação, entre eles 2/8 (25\%) que estavam recebendo infusão contínua de lidocaína e $1 / 8(12,5 \%)$ que estava recebendo infusão contínua de fentanil.
O agente halogenado sevofluorano foi utilizado em dois pacientes submetidos à hemilaminectomia e ambos apresentaram bradicardia no período transoperatório (Tabela 5). Nos animais que receberam fentanil, ocorreu bradicardia em um cão (1/6) $(16,66 \%)$ e nos animais que receberam lidocaína um animal foi a óbito $(1 / 7)(14,29 \%)$ e dois animais apresentaram bradicardia (2/7) $(28,57 \%)$.

Tabela 5. Agentes inalatórios halogenados utilizados em cães e gatos submetidos à neurocirurgia no Hospital Veterinário da UEL e as complicações observadas durante o procedimento anestésico, Londrina, 2007.

\begin{tabular}{|c|c|c|c|c|c|c|c|c|c|c|c|c|}
\hline \multicolumn{13}{|c|}{ Halogenados utilizados em diferentes neurocirurgias } \\
\hline \multirow[t]{2}{*}{ Complicações } & \multicolumn{3}{|c|}{$\mathrm{H}$} & \multicolumn{2}{|r|}{$\mathrm{S}$} & \multicolumn{2}{|c|}{$\mathrm{E}$} & \multicolumn{2}{|l|}{$\mathrm{C}$} & \multicolumn{3}{|c|}{ Total } \\
\hline & Hal & Iso & Sev & Hal & Iso & Hal & Iso & Hal & Sev & Hal & Iso & Sev \\
\hline Bradicardia & 6 & 6 & 2 & - & - & - & - & 1 & - & 7 & 6 & 2 \\
\hline Óbito & - & - & - & - & 2 & - & - & - & - & - & 2 & - \\
\hline Total de animais com complicações & $6(43 \%)$ & $6(23 \%)$ & $2(100 \%)$ & - & $2(50 \%)$ & - & - & $1(100 \%)$ & - & $7(36,8 \%)$ & $8(25 \%)$ & $2(66,7 \%)^{*}$ \\
\hline Total de animais anestesiados & 14 & 26 & 2 & 2 & 4 & 2 & 2 & 1 & 1 & 19 & 32 & 3 \\
\hline
\end{tabular}

H: Hemilaminectomia; S: "Slot”Cervical; E: Estabilização vertebral; C: Craniotomia

* Não houve diferença significativa $(\mathrm{p}=0,094)$ entre os tipos de halogenados e presença de complicações. 
Não ocorreu óbito com a utilização de halotano, porém nas anestesias com o agente isofluorano dois animais vieram a óbito: um animal submetido à técnica de slot cervical não retornou à respiração espontânea após o término da anestesia e outro, também submetido à mesma técnica, que apresentou hemorragia grave do plexo venoso vertebral interno no período transcirúrgico.

Através da análise estatística constatou-se que não houve associação entre a infusão contínua de fármacos analgésicos administrados durante o transoperatório e as complicações observadas (bradicardia e óbito, $\mathrm{p}=1,0$ ) (Tabela 4), e também não houve associação entre os agentes halogenados empregados e as complicações visualizadas durante o procedimento anestésico $(\mathrm{p}=0,094)$ (Tabela 5).

\section{Discussão}

A duração média da anestesia foi semelhante entre os pacientes que apresentaram complicações e os que não apresentaram. Muitas complicações ocorrem devido ao tempo anestésico prolongado (MUIR, 1996). Entretanto, através da análise estatística o tempo anestésico não foi relacionado com a ocorrência de complicações.

Optou-se pela utilização de levomepromazina como medicação pré-anestésica em 16 animais devido ao temperamento particular destes, com o intuito de diminuir a ansiedade sem promover estado de sedação (CORTOPASSI; FANTONI, 2002). Porém segundo Court et al. (1990a) a medicação pré-anestésica (MPA) no paciente neurológico não deve afetar a função cardiorrespiratória e os fenotiazínicos devem ser usados cuidadosamente devido ao risco de hipotensão.

Os pacientes com lesão medular-vertebral sentem bastante dor e freqüentemente desenvolvem arritmias. A MPA realizada com opióide, associada a um agente anticolinérgico é benéfica para redução da dor pré e pós-operatória em pacientes submetidos à cirurgia vertebral (SILVA et al., 2006), porém a sedação profunda é contra indicada, especialmente em animais com lesões medulares traumáticas, visto que pode ocasionar diminuição do tônus muscular e remoção do efeito protetor da musculatura epaxial sobre as vértebras instáveis (SILVA et al., 2006). Os efeitos dos opióides no fluxo sangüíneo cerebral e na pressão intracraniana são mínimos, porém pode indiretamente ocorrer aumento na pressão intracraniana devido à hipercapnia decorrente da hipoventilação induzida pela administração de opióides (HARVEY; GREENE; THOMAS, 2007).

A indução anestésica foi realizada com propofol em 57,4\% dos casos, por ser um agente hipnótico intravenoso de curta ação, que proporciona uma indução rápida e sem sinais de excitação (GLOWASKI; WETMORE, 1999), além de diminuir a pressão intracraniana e o fluxo sangüíneo cerebral, úteis em lesões encefálicas (BRANSON, 2007). Em 37,03\% dos animais optou-se pela associação do propofol com benzodiazepínicos, realizando uma anestesia balanceada, indicada por não causar depressão da função cardiovascular e respiratória (FANTONI; CORTOPASSI, 2002). O tiopental, escolhido na indução de um animal submetido à técnica de "slot" cervical e outro submetido à craniotomia, tem efeito protetor e anticonvulsivante em pacientes que sofreram trauma medular e encefálico, mas em virtude dos efeitos depressores no sistema cardiorrespiratório e no tronco encefálico deve ser usado com cautela (SILVA et al., 2006; BRANSON, 2007).

O objetivo da infusão contínua de fármacos anestésicos durante o período transoperatório foi minimizar o requerimento de anestésicos voláteis, limitando seus prejuízos cardiovasculares (PYPENDOP; ILKIW, 2005). Segundo Valverde et al. (2004), a lidocaína em infusão contínua reduz a concentração alveolar mínima de maneira dosedependende e não induz clinicamente alterações significantes na freqüência cardíaca e pressão arterial. No presente estudo, dois animais que estavam recebendo infusão contínua de lidocaína e um animal com infusão contínua de fentanil 
apresentaram bradicardia, não havendo relação estatisticamente significante entre o uso destes fármacos e a complicação observada. McEwan et al. (1993) e Brunner et al. (1994) relatam diminuição da concentração alveolar mínima do isofluorano na ordem de 50\% com fentanil. Fantoni et al. (1999) relatam que a administração intravenosa de analgésicos opióides, como o fentanil possibilitou o emprego de menores concentrações de halotano, promovendo anestesia estável com manutenção da pressão arterial dentro dos valores normais para a espécie canina.

Embora na análise estatística não tenha sido constatado associação entre os agentes inalatórios e as complicações observadas, estas ocorreram com maior frequência com o halotano do que com o isofluorano. Segundo Seim III (2005), os efeitos cardiovasculares causados pelo isofluorano são mínimos, havendo manutenção do débito cardíaco sem sensibilização do miocárdio às catecolaminas.

A principal complicação observada neste estudo foi a bradicardia nos animais submetidos à hemilaminectomia toracolombar. A ventilação controlada pode ter colaborado para a ocorrência de bradicardia por diminuir o débito cardíaco ao ocasionar diminuição de pressão negativa intratorácica sobre os grandes vasos no mediastino, comprometendo o retorno venoso (SILVA et al., 2006). Segundo estudo de Stauffer et al. (1988), que compararam pacientes submetidos à descompressão cervical com pacientes submetidos à descompressão torácica e lombar, houve risco de arritmia 2,5 vezes maior em cães submetidos à cirurgia cervical do que a laminectomia toracolombar. A bradicardia é tolerável, desde que moderada e rítmica, pois durante a anestesia profunda ocorre queda no metabolismo basal (MASSONE, 2003).

Em geral, quanto mais cranial a lesão medular (cervicotorácica), maior a chance de ocorrer dificuldade na manutenção da estabilidade cardiovascular e respiratória (BREARLEY; WALSH, 1999), provavelmente porque as lesões medulares interrompem as conexões de tratos nervosos simpáticos advindos do tronco encefálico, diminuindo as funções motoras cardiovasculares (SEIM III, 2005).

Nas craniotomias os anestésicos utilizados foram halotano ou sevoflurano, ocorrendo bradicardia no animal anestesiado com halotano. $\mathrm{O}$ halotano não é o anestésico mais indicado em animais com trauma cranioencefálico por elevar a pressão intracraniana (PIC) (SILVA et al., 2006), entretanto foi utilizado neste caso para retirada de projétil balístico localizado superficialmente em ossos do crânio, pois na avaliação pré-anestésica não havia sinais de aumento da PIC. O uso do sevofluorano foi priorizado no paciente pediátrico, pois o mesmo estava debilitado. Além disso, este agente não possui potencial convulsivo (SILVA et al., 2006), porém neste estudo retrospectivo o número de animais anestesiados com sevofluorano não foi significativo para que pudesse ser melhor avaliado. O isofluorano, por diminuir a taxa metabólica cerebral com menor efeito sobre o fluxo sangüíneo cerebral (FSC) seria o agente ideal para pacientes com aumento da pressão intracraniana (CORNICK, 1992; SILVA et al., 2006).

A afecção neurológica e o próprio procedimento cirúrgico podem causar alterações cardiovasculares. Os dois animais que vieram a óbito estavam sendo submetidos à técnica de "slot" cervical. Segundo Sharp e Wheller (2006), os pacientes tetraplégicos freqüentemente possuem comprometimento respiratório subclínico e necessitam de suporte ventilatório durante a cirurgia. Esses pacientes geralmente desenvolvem dificuldade respiratória devido à diminuição no período trans e póscirúrgico da função dos músculos diafragmáticos e intercostais. Um dos animais não retornou a ventilação espontânea após o término do procedimento cirúrgico, provavelmente porque as lesões medulares, especialmente aquelas craniais ou nos segmentos medulares cervicais caudais, podem interromper conexões entre o centro respiratório e a origem medular dos nervos frênico e torácicos, 
levando à paresia ou paralisia respiratória (SEIM III, 2005). O outro cão veio a óbito devido à hemorragia intensa do plexo venoso vertebral interno. Segundo a literatura consultada isto pode ocorrer nesta técnica, o que pode ser fatal como observado no presente trabalho (COURT et al., 1990b. Nestes casos, os agentes anestésicos empregados não foram responsáveis pelas complicações observadas.

Não foi possível avaliar alterações no padrão respiratório, pois $43(79,63 \%)$ dos 54 animais anestesiados foram ventilados mecanicamente. A ventilação controlada foi essencial nos procedimentos, pois evitou hipercapnia e hipóxia por hipoventilação, porém pode ter colaborado para a bradicardia (SILVA et al., 2006).

A complicação mais observada neste estudo foi bradicardia e o isofluorano foi o agente que apresentou a menor taxa de complicações. $\mathrm{O}$ conhecimento dos efeitos dos fármacos, das técnicas anestésicas, das doenças neurológicas e das alterações que podem ocorrer concomitantemente é essencial para evitar alterações no sistema nervoso central, causadas pelos fármacos, pela doença ou associação destes fatores.

\section{Conclusão}

Os principais protocolos utilizados foram propofol para indução e halotano ou isofluorano para manutenção da anestesia. Estes protocolos mantiveram o plano anestésico desejado para o procedimento cirúrgico e não causaram complicações durante o período anestésico em $37 / 54$ animais $(68,5 \%)$. Entre as complicações observadas, $7 / 19(36,8 \%)$ ocorreram em pacientes submetidos à anestesia com halotano e 8/32 (25,2\%) em pacientes anetesiados com isofluorano. A principal complicação neste estudo foi bradicardia, que acometeu 15/54 (27,8\%) dos pacientes.

\section{Agradecimento}

A professora Dr. Roberta Lemos Freire pelo auxílio na análise estatística do trabalho.

\section{Referências}

BRANSON, K. R. Injectable and alternative anesthetic techniques. In: THURMON, J. C.; TRANQUILLI, W. J.; GRIMM, K. A. Lumb \& Jones'veterinary anesthesia. 4. ed. Iowa: Blackwell Publishing, 2007. p. 273-299.

BREARLEY, J. C.; WALSH, K. Neurological disease. In: SEYMOUR, C.; GLEED, R. Manual of small animal anaesthesia and analgesia. 2. ed. Cheltenham: British Small Animal Veterinary Association, 1999. p. 231-236.

BRUNNER, M. D.; BRAITHWAITE, P.; JHAVERI, R.; MCEWAN, A. I.; GOODMAN, D. K.; SMITH, L. R.; GLASS, P. S. MAC reduction of isofluorane by sufentanil. British Journal of Anaesthesia, London, v. 72, n. 1, p. 42-46, 1994.

CORNICK, J. L. Anesthetic management of patients with neurologic abnormalities. Compendium: Continuing Education for Veterinarians, Yardley, v. 14, n. 2, p. 163170, 1992.

CORTOPASSI, S. R. G.; FANTONI, D. T. Medicação pré-anestésica. In: . Anestesia em cães e gatos.

São Paulo: Roca, 2002. p. 280-285.

COURT, M. H.; DODMAN, N. H.; NORMAN, W. M.; SEELER, D. C. Anaesthesia and central nervous system disease in small animals part I: general considerations. British Veterinary Journal, London, v. 146, n. 3/4, p. 285-295, 1990a.

. Anaesthesia and central nervous system disease in small animals part II: anaesthetic management for specific diseases and procedures. British Veterinary Journal, London, v. 146, n. 3/4, p. 295-308, 1990 b.

CRUZ, M. L. Anestesia em ortopedia. In: FANTONI, D. T.; CORTOPASSI, S. R. G. Anestesia em cães e gatos. São Paulo: Roca, 2002. p. 280-285.

FANTONI, D. T.; AMBROSIO, A. M.; FUTEMA, F.; MIGLIATI, E. R.; TAMURA, E. Y. Utilização de alfentanil, sulfentanil e fentanil em cães anestesiados com halotano. Ciência Rural, Santa Maria, v. 29, n. 4, p. 681-688, 1999.

FANTONI, D. T.; CORTOPASSI, S. R. G. Conceitos em Anestesiologia. In: . Anestesia em cães e gatos. São Paulo: Roca, 2002. p. 280-285. 
GLOWASKI, M. M.; WETMORE, L. A. Propofol: application in veterinary sedation and anesthesia. Clinical Techniques in Small Animal Practice, Philadelphia, v. 14, n. 1, p. 1-9, 1999.

HARVEY, R. C.; GREENE, S. A.; THOMAS, W. B. Neurological disease. In: THURMON, J. C.; TRANQUILLI, W. J.; GRIMM, K. A. Lumb \& Jones' veterinary anesthesia. 4. ed. Iowa: Blackwell Publishing, 2007. p. 903-913.

KOBRINE, A. I.; DOYLE, T. F.; RIZZOLI, H. V. Spinal cord blood flow as affected by changes in systemic arterial blood pressure. Journal of Neurosurgery, Charlottesville, v. 44, n. 1, p. 12-15, 1976.

MASSONE, F. Anestesiologia veterinária. 4. ed. Rio de Janeiro: Guanabara Koogan, 2003.

McEWAN, A. I.; SMITH, C.; DYAR, O.; GOODMAN, D.; SMITH, L. R.; GLASS, P. S. Isofluorane minimum alveolar concentration reduction by fentanil. Anesthesiology, Philadelphia, v. 78, n. 5, p. 864-869, 1993.

MUIR, W. W. Considerations for general anesthesia. In: THURMON, J. C.; TRANQUILLI, W. J.; BENSON, G. J. Lumb \& Jones'veterinary anesthesia. 3. ed. Baltimore: Wolters Kluwer, 1996. p. 7-30.

PYPENDOP, B. H.; ILKIW, J. E. The effects of intravenous lidocaine administration on the mimimum alveolar concentration of isoflurane in cats. Anesthesia \& Analgesia, Baltimore, v. 100, n. 1, p. 97-101, 2005.

SEIM III, H. B. Cirurgia cerebral. In: FOSSUM, T. W. Cirurgia de pequenos animais. 2. ed. São Paulo: Roca, 2005. p. 1313-1329.
SEIM III, H. B. Surgery of the cervical spine. In: FOSSUM, T. W. Small animal surgery. 2. ed. Saint Louis: Mosby, 2002. p. 1213-1214.

SHARP, N. J. H.; WHEELER, S. J. Small animal spinal disorders diagnosis and surgery. 2. ed. Edinburgh: Elsevier Mosby, 2006.

SILVA, S. R. A. M.; NÓBREGA NETO, P. I.; TUDURY, E. A.; FANTONI, D. T. Anestesia de cães e gatos com distúrbios neurológicos. Clínica Veterinária, São Paulo, v. 11, n. 64, p. 34-46, 2006.

STAUFFER, J.; GLEED, R. D.; SHORT, C. E.; SCHUKKEN, Y. H. Cardiac dysrhythmias during anesthesia for cervical descompression in the dog. American Journal of Veterinary Research, Chicago, v. 49, n. 7, p. 1143-1146, 1988.

STEFFEY, E. P. Inhalation anesthetics. In: THURMON, J. C.; TRANQUILLI, W. J.; BENSON, G. J. Lumb \& Jones' veterinary anesthesia. 3. ed. Baltimore: Wolters Kluwer, 1996. p. 297-330.

TORRES, M. L. A.; BONASSA, J. Princípios básicos da ventilação controlada. In: FANTONI, D. T.; CORTOPASSI, S. R. G. Anestesia em cães e gatos. São Paulo: Roca, 2002. p. 95-105.

VALVERDE, A.; DOHERTY, T. J.; HERNANDEZ, J.; DAVIES, W. Effect of lidocaine on the minimum alveolar concentration of isofluorane in dogs. Veterinary Anaesthesia and Analgesia, Oxford, v. 31, n. 4, p. 264271, 2004. 
